\title{
Effective tocotrienol dosage traceability system using blockchain technology
}

\author{
Norhaslinda Kamaruddin ${ }^{1}$, Abdul Wahab ${ }^{2}$ \\ ${ }^{1}$ Advanced Analytics and Engineering Centre, Faculty of Computer and Mathematical Sciences, \\ Universiti Teknologi MARA, Malaysia \\ ${ }^{1}$ Fakultas Sains dan Teknologi, Kampus C, Universitas Airlangga, Indonesia \\ ${ }^{2}$ Kulliyah of Information and Communication Technology, International Islamic University Malaysia, Malaysia
}

\begin{tabular}{l}
\hline \hline Article Info \\
\hline Article history: \\
Received Dec 17, 2019 \\
Revised Feb 23, 2020 \\
Accepted Apr 24, 2020
\end{tabular}

\section{Keywords:}

Big data analytics

Blockchain

Internet of Things

Tocotrienol

Traceability system

\begin{abstract}
Tocotrienol dosage, especially in vitamin E, is important for treatment and prevention of diseases. To date, the dosage is given based on the physician's knowledge and experience to suit the patient's needs. The alteration of the dosage is depending on the way the patient's body reaction and coping mechanism which is different from one to another. Hence, the optimal dosage is very difficult to achieve and may result in undesirable side effects. An alternative solution using blockchain technology to trace and chart the dosage of tocotrienol is proposed to capture the effective measure for the patient. With the advancement of the internet of things (IoT) and big data analytics technologies, an effective tocotrienol dosage is possible by utilizing the data gathered from the individual patient for tocotrienol dosage personalization profiling. Then, the output can be used to assist the physician to diagnose an appropriate amount of tocotrienol dosage for optimum effect. This paper discusses the theoretical framework of using blockchain technology to develop an effective tocotrienol dosage traceability system. It is envisaged that such an approach can be a guide to the health practitioners to administer the correct dosage for the patient and subsequently leads to a better quality of life.
\end{abstract}

This is an open access article under the CC BY-SA license.

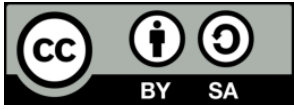

\section{Corresponding Author:}

Norhaslinda Kamaruddin,

Advanced Analytics and Engineering Center (AAEC),

Universiti Teknologi MARA,

40450 Shah Alam, Selangor, Malaysia.

Email: norhaslinda@tmsk.uitm.edu.my

\section{INTRODUCTION}

Recent trends in big data analytics have seen tremendous growth in many sectors especially for financial [1,2] and medical sectors [3,4] by using artificial intelligence (AI) in analyzing a huge amount of data. One of the prime concerns in both applications is the traceability of information especially in the medical sector where a lot of insights can be gleaned based on the medications and treatments provided. This information can improve and expedite the intervention processes with a higher confidence level and a better probability of success. In this paper, we are proposing the tocotrienol dosage traceability system using blockchain technology where the variability of individual patient dosage is very high and requires close intervention.

There have been many occasions where vitamin E was used to treat and prevent heart and blood vessel diseases, heart attack, chest pain, leg pain due to blocked arteries, and high blood pressure. In some cases, vitamin E was also used to treat diabetic patients with complications, as well as various types 
of cancer; such as; lung and oral cancer, gastric, prostate, and pancreatic. Vitamin E is also applied to the skin to protect against the effects of the chemicals and to slow down the effect of aging. The usage of vitamin $\mathrm{E}$ is boundless; therefore, researchers continue to experiment with its usage for various kinds of diseases and enhancements.

Most dietician recommends eating high fiber and complex carbohydrates food in fruits, vegetables and whole grains rather obtaining antioxidants, including vitamin $\mathrm{E}$ from supplements until more is known about the risks and benefits of taking supplements [5]. Unfortunately, producers of foods have been using vitamin $\mathrm{E}$ as gimmicks in many food products including cereals, vegetable oils, and eggs. Surprisingly, not many consumers know the importance of the absorption rate of vitamin $\mathrm{E}$ in the body, which is critical to ensure the usefulness of vitamin $E$ for a treatment. In its natural form, vitamin $E$ exists in eight different forms namely: $\alpha-, \beta-, \gamma$ - and $\delta$-tocopherol; and $\alpha-, \beta-, \gamma$-, and $\delta$ - tocotrienol. Shahidi and Ambigaipalan [6] reported that less than $1 \%$ of vitamin $\mathrm{E}$ is related to tocotrienols. However, dietician tends to neglect the nontocopherol vitamin $\mathrm{E}$ molecules to be researched into [6] because $\alpha$-tocopherol readily exists in the human body and it is comparably efficient to use any vitamin E molecules as antioxidants.

Recently, researchers have been seriously looking into the non-tocopherol molecules which seem to have a greater impact in processing anti-cancer and cholesterol-lowering properties [7]. Some of the experimental results indicated that members of the vitamin E family are not redundant to their biological functions [8]. It is found that $\alpha$-, $\gamma$-, and $\delta$ - tocotrienols of the vitamin $E$ molecules have special functions in health that are very different from $\alpha$-tocopherol. It is noted that the $\alpha$-tocotrienol managed to avert neurodegeneration in a concentrated form [9]. This indicates that any form of natural vitamin $E$ molecule does have the potential of all biological functions and is functionally unique. Hence, the evidence of toxicity in the tocopherol molecule due to excess dosage is irrelevant to be linked with tocotrienols [10]. Further research must be investigated the toxicity of tocotrienols separately as similarity of tocopherol and tocotrienols functionality do not exist in high dosage into the lesser-known forms of vitamin E. Such detail studies can help better selection of appropriate vitamin E molecule for in addressing unique used of vitamin E supplements [11].

Certain processes in palm kernel oil can extracts up to an average of $70 \%$ tocotrienols to allow a higher or tocotrienol-rich fraction (TRF) palm kernel oil, although all eight molecules of the tocotrienols and tocopherols exist [11]. In the early days, researchers were looking at the $\alpha$-tocopherol [12] but recently tocotrienols have attracted a large number of researchers to look into due to differing functionality and properties of tocotrienols to that of tocopherols by having an unsaturated side chain [13] resulting in significantly different biological activities. The extraction process parameters of the enhanced TRF in the palm kernel oil plant can be adjusted to optimize the ratio of tocotrienols to tocopherols contents. It has shown that improvement between $10 \%$ to $20 \%$ can already be achieved by moving the process parameters, thus improvements and optimization of the ratio between tocotrienols and tocopherols can be achieved in vitamin E supplement [14]. Some clinicians use different types of carriers like the medium-chain triglycerides (MCT) to further improve the ratio of tocotrienols. In lipid-based drugs, MCT has been used as an absorption enhancer that can be obtained in coconut and palm kernel oil [15].

Hence, a complex system is needed to manage and analyze a large amount of data and information from the human to administer such complex supplements of tocotrienols for it to perform better and meeting all the requirements without compromise. As indicated earlier, it is important to be able to adjust the process parameters through a better understanding of the process variation in detail when extracting the tocotrienols. Such a system must also be able to provide traceability of the manufacturer producing the vitamin E so that amount of concentration of tocotrienols or tocopherols can be known at any point in time. To date, the usage of vitamin E especially tocotrienol is still not fully explored and requires the profile of the patient to optimize the dosage of tocotrienols. The dynamic repository system is required to ensure proper tracking of the trial that has been carried out to optimize dosage effectively [16]. Profiling each patient and traceability of tocotrienol dosage and the source of tocotrienol including manufacturing details will be required to ensure accurate and genuine information. Such a system requires the use of big data analytics on blockchain for traceability. Presently, the availability of such an integrated system may not be easily purchased and put together, thus we propose the blockchain cloud system (BcCS) for tracking and analyzing the dosage of tocotrienols trials.

In this paper, we study the utilization and development situation of blockchain technology and analyze the advantages and disadvantages of using blockchain technology in building the tocotrienols optimizing traceability system. Such a system would effectively guarantee the repeatability and dosage accuracy by gathering, transferring, and sharing the authentic data of the patients and manufacturing details in production, processing, warehousing, distribution, and selling links. 


\section{LITERATURE REVIEW}

Tocotrienols are the major interest of recent research for various biological potentials including anticancer activity. Although tocotrienols have four isomers in the vitamin $\mathrm{E}$ group of compounds, there are also another four isomers that belong to tocopherols. Most of the tocotrienols are obtained from various natural sources including palm oil, rice bran oil, and annatto. However, due to the small amount of tocotrienols in its natural form, a large amount is required to be consumed before it can achieve the desired therapeutic effects. Besides, due to the poor absorption and distribution of the tocotrienols through the gut, it is impossible to achieve the desired therapeutic amount directly from its natural sources. An alternative for a tocotrienol carrier is needed for body consumption.

\subsection{Extraction of tocotrienols}

Many studies on humans and animals have shown the great potential of using tocotrienols for various medical conditions namely; cancer, inflammation, and neuroprotection as vitamin E supplements. Tocotrienols are lipophilic thus can be absorbed easily through food and with the aid of the different type of carriers can help the absorption of tocotrienol to the individual body system. The efficiency of tocotrienol absorption has been a recent study due to the medical benefits that can gain from tocotrienols [12].

Researchers recently found that other molecules of the vitamin E of the tocotrienols namely the $\gamma-\mathrm{T}$, $\delta$-T, and $\gamma$-T3 have a special type of antioxidant and anti-inflammatory properties that are far better off than the $\alpha$-tocopherol in combating chronic diseases. Efficient penetration into tissues consisting of saturated fatty layers like the brain and liver can use tocotrienols in the form of an unsaturated chain. Biodistribution studies in the accumulation of tocotrienols in the individual organs of the body are still inadequate and require further investigation. There has been a wide range of tocotrienol studies and its benefits against some human ailments with promising potential especially in the area of anti-aging, which deserves further investigation into these lesser-known forms of vitamin E [9]. Many natural compounds found in wheat germ, vegetable oils, barley, and certain types of nuts can be a good source to extract tocotrienols. Meganathan et al. [17] had found that using an equivalent TRF, and gamma and delta tocotrienols (GDT) of $800 \mathrm{mg}$ and $600 \mathrm{mg}$ respectively on healthy participants were not bioequivalent. TRF compare to GDT seems to produce less gamma-tocotrienol [17] and generally seems to be safe and tolerated by all the participants. Thus, it is important to look at the detailed dosage of each tocotrienols factions to ensure effectiveness in terms of bioequivalent.

\subsection{Traceability of food chain and its equivalent}

In another study, radio frequency ID (RFID) coupled with blockchain technology was used in developing the agri-food supply chain management system for traceability. Tian listed down the advantages and disadvantages of such a system, which can be costly for a small tracking system [18]. Such a traceability system can ensure that information stored in the blockchain cannot be easily erased or corrupted by any individual due to the distributed ledger technology implemented in blockchain, thus ensuring data integrity and security. Moreover, Biswas uses the blockchain supply chain system to track wines in the global market for counterfeiting, transparencies, and security [15]. Since there has been an increase in counterfeit products in the global market, blockchain is an effective solution to overcome such a problem. The blockchain technology enables verification without any dependencies to any third-party applications. The data cannot be deleted or amended and the data structure in blockchain only allows the append process. It makes the data stable and traceable. It can lead to the point of origin if irregularities are yielded. Moreover, the transactions are verified in every single time they are passed from one blockchain node to another making it least prone to human error and protect the data from any possible tempering.

However, blockchain technology suffers from several drawbacks. One of the disadvantages of blockchain technology is the transaction speed for some applications such as bitcoin may not be as fast as expected [19]. For example, to date, a bitcoin transaction needs approximately 40 minutes on average for verification purposes [20]. Privacy is another critical issue since neither company nor individuals would like to publish all information onto a public database. However, private blockchains with cryptographic schemes can be used to efficiently address these issues. In a private blockchain, sensitive information can be kept secret by encrypting them using a pre-distributed secret key whereas public information can be stored in plain text. In addition, to address transaction speed issue, the number of miners can be pre-selected in a private blockchain-based system where all these miners are assumed to have some level of trust.

Blockchain technology based on the distributed ledger technology will be keeping track and collecting all the consent and consolidate it. All data related to consent is like a fingerprint that can be verified and is part of the integral system. Thus, integrity and consistency of data from both the stakeholders' and patients' perspectives are transparent and visible to whichever parties having accessibility and hence control over their consent [21].

In Galvez's work [22], tracking and authenticating the food supply chain provides a detailed understanding of the criticality of traceability. Issues on traceability and ensuring transparency can be easily 
achieved using blockchain. This growing disruptive technology with the Internet of Things (IoT) suggests that researchers look into blockchain technology more seriously and seems to be able to provide help to achieve traceability in all cases or scenarios. Resius [23] addressed meaningful questions to foster substantial blockchain research by identifying several future research potentials given the breadth of open questions he manages to show where research can benefit from multidisciplinary collaborations and presents data sources as starting points for empirical investigations.

Subsequently, Chen [20] proposes a novel approach called an artificial neural network (ANN) based on Takagi-Sugeno fuzzy (TSF) cognitive maps also has a traceability chain algorithm and the use of artificial intelligence in many human-related applications [24-26]. Blockchain mining is used to evaluate and optimize the decision-making process. Objective functions for optimized decision computation is described as participant nodes constraint method. The study succeeds in meeting the reduction of mining efforts for the traceability chain being processed. His findings also provide a preliminary indication of deep learning applied big blockchain transactions data [20]. Nonetheless, it requires heavy computation time and with the blockchain validation, Chen's system may not work with a large number of users, as it may take a long time for each validation to takes place.

In summary, blockchain has been used for traceability in many forms especially where privacy is required in a public environment. Even the application of public blockchain for a private application can be easily implemented with sufficient accuracy due to the cryptographic nature of blockchain and the distributed ledger of the blockchain. In fact, blockchain technology has been used for verifications, validations, authentications, traceability, and many more applications yet to be explored. Furthermore, blockchain can produce a robust method in tracking products for transportation needs when coupled together with IoT and RFID. In the next section, we propose the use of blockchain for optimizing the dosage of tocotrienol for the effective intervention of disease for individuals.

\section{THE BLOCKCHAIN CLOUD TRACEABILITY SYSTEM}

There has been much research into blockchain traceability and seems to provide key advantages rather than using RFID or IoT alone [27, 28]. In fact, a combination of RFID or IOT together with blockchain traceability can provide an ideal solution to many systems that require tracking of things. This concept is like the supply chain management system (SCM) where it is important to track the incoming parts of the manufacturing processes to ensure smooth production flow. Figure 1 shows the block diagram of the blockchain traceability tocotrienols laboratory trial system where it requires the tocotrienols blockchain on the top portion and the patients profiling blockchain at the bottom portion. Both require separate blockchain servers to ensure the privacy of the information as there may be a need to have private blockchain.

\subsection{Tocotrienols production chain (TPC)}

The tocotrienols blockchain requires the manufacturer of the tocotrienols production to initiate the entry of the detail of tocotrienol being produced. All information about the tocotrienols production that can help to track the tocotrienols will be critical. In this case, information, such as; manufacturing date, the producer of tocotrienol, the origin of the tocotrienol, the natural source of vitamin $\mathrm{E}$ and the process of extractions. All the detailed process information provides us a detailed amount of tocotrienols that can be extracted including lab test results of the final production for an individual batch of tocotrienol produced, including date and time-stamped.

Once the wholesaler received the tocotrienols, the batch will then be verified and updated in the blockchain nodes before the tocotrienols are sent to the pharmacist. Once the pharmacist received the tocotrienols, the verification, and update processes are conducted to the tocotrienols blockchain nodes. This step is to ensure that the pharmacist also knows the product is at hand and he/she has taken the necessary action to verify the product, either through inspection or some other test methods. The pharmacist then delivers the tocotrienols to the researchers to be used for the patient. Such traceability is important and critical so that effectiveness of the dosage and delivery of the tocotrienol to the patient can be monitored accurately and if any tracking is required, it can be traced all the way to the patient and the production processes. Finally, the researchers know exactly where the tocotrienol comes from and when he administers the supplements and the amount of tocotrienol that should be given.

\subsection{Patients profiling chain (PPC)}

On the bottom part of Figure 1 shows the patient profiling chain where each patient will enter all their personal detail and verified by medical practitioner especially information about the drugs taken and the symptoms suffered. Besides, the various lab test data, such as; blood test, X-ray, MRI, fMRI 
and other laboratory test results need to be entered. This information will be also be updated in the blockchain nodes and verified by the medical practitioners and/or the laboratory personnel. The researchers will then verify all this information and match with the tocotrienols that the researcher administers to the patient. Patient information will be updated as and when new tocotrienols are administered or any test is carried out by the laboratory. Such a system is comprehensive enough to now allow the research to analyze in detail the effectiveness of tocotrienols administered for each patient.



Figure 1. Blockchain traceability block diagram for tocotrienol laboratory trials

\subsection{Tocotrienols-patient analysis}

Now that all the information details are already available in the TPC and the PPC, the researchers can fully analyze the effectiveness of the tocotrienols in improving the patient condition thus allowing 
the researcher to increase or reduce the dosage or change to other vitamins $\mathrm{E}$ for more effective intervention. Such trials in big data analytics allow researchers to understand the performance of different vitamin $\mathrm{E}$ to handle different diseases with a different patient profile. Patients with a similar profile and similar disease can use the same amount of dosage and vitamin $\mathrm{E}$ to be administered for effective intervention. This will help speed up intervention and cost-effective. Whenever a new product is available, then a similar process can help expedite researchers to understand the effectiveness of the intervention.

In addition, a similar AI approach can also be used to analyze and visualize the result of the trend of the dosage for the tocotrienols. Such an approach can be utilized for a personalized report that can be extended in many domains [29-31]. It is hoped with such a system it can help researchers and physicians to administer the optimal dosage to the patient for the first time rather than the trial and error approach which may have undesirable side effects to the patients.

\section{SUMMARY AND CONCLUSION}

We have been using the NEM distributed ledger technology (blockchain), which is a public, open blockchain system, for our research in various applications and has shown potential to be used especially for traceability. The NEM system seems to be faster and compact, which is ideal for traceability. It has been used for tracking antiques and many confidential products either on private or public blockchain even with minimal nodes. Although blockchain only stores text information which will be used to verifies the owner of the data thus enabling access to information and images for detailed analysis. The blockchain technology is suitable to track and ensure the data in the nodes are updated and are not easily tempered. Such an approach allows a highly traceable audit trail that can be used to monitor the changes in tocotrienol dosage administered by the researchers for the optimal output. Subsequently, with the realization of such a system, it is envisaged that the quality of life of the patients can be also improved.

\section{ACKNOWLEDGEMENTS}

The authors would like to thank Institute of Quality and Knowledge Advancement (InQKA), University Teknologi MARA for providing financial support to publish the work and Faculty of Computer and Mathematical Sciences, Universiti Teknologi MARA (UiTM), International Islamic University Malaysia (IIUM) and Ministry of Higher Education Malaysia (MOHE) for data collection through the MITRA grant (600-IRMI/PERDANA 5/3/MITRA (007/2018)-3) to conduct the work published in this paper.

\section{REFERENCES}

[1] T. Hatch, "The impact of Big Data on finance," Strategic Finance 101, no. 8, pp. 46-52, 2020.

[2] R. Yang, L. Yu, Y. Zhao, H. Yu, G. Xu, Y. Wu, and Z. Liu, "Big data analytics for financial market volatility forecast based on support vector machine," Int. Journal of Information Management, vol. 50, pp. 452-462, 2020.

[3] N. Mehta, A. Pandit, and M. Kulkarni, "Elements of healthcare Big Data analytics," Big Data Analytics in Healthcare, Springer, Cham., pp. 23-43, 2020.

[4] W. Raghupathi and V. Raghupathi, "Big data analytics in healthcare: promise and potential," Health Information Science and Systems, vol. 2, no. 1, pp. 1-10, 2014.

[5] R. E. Olsen, "Vitamin E and its relation to heart disease," Circulation, vol. 48, no. 1, pp. 179-184, 1973.

[6] F. Shahidi and P. Ambigaipalan, "Phenolics and polyphenolics in foods, beverages and spices: Antioxidant activity and health effects-a review," Journal of Functional Foods, vol. 18, part B, pp. 820-897, October 2015.

[7] C. K. Sen, S. Khanna, and S. Roy, "Tocotrienols: Vitamin E beyond tocopherols," Life Sciences, vol. 78, no. 18, pp. 2088-2098, March 2006.

[8] M. Matringe, B. Ksas, P. Rey, and M.Havaux, "Tocotrienols, the unsaturated forms of vitamin E, can function as antioxidants and lipid protectors in tobacco leaves," Plant Physiology, vol. 147, no. 2, pp. 764-778, 2008.

[9] C. K. Sen, C. Rink, and S. Khanna, "Palm oil-derived natural vitamin E $\alpha$-tocotrienol in brain health and disease," Journal of the American College of Nutrition, vol. 29, no. Sup3, pp. 314S-323S, 2010.

[10] C. K. Sen, S. Khanna, C. Rink, and S. Roy, "Tocotrienols: The emerging face of natural vitamin E," Vitamins and Hormones, vol. 76, pp. 203-261, 2007.

[11] F. Shahidi and A. C. De Camargo, "Tocopherols and tocotrienols in common and emerging dietary sources: occurrence, applications and health benefits," Int. Journal of Molecular Sciences, vol. 17, no. 10, pp. 1-29, 2016.

[12] M. I. Kabir, M. Adnan, and M. M. Rahman, "Natural sources of tocotrienols: A note on absorption," Journal of In Silico \& In Vitro Pharmacology, vol. 3, no. 20, pp. 1-5, 2017

[13] H. Ahsan, A. Ahad, and W. A. Siddiqui, "A review of characterization of tocotrienols from plant oils and foods," Journal of Chemical Biology, vol. 8, no. 2, pp. 45-59, 2015.

[14] C. Zhang, R. E. Cahoon, S. C. Hunter, M. Chen, J. Han, and E. B. Cahoon, "Genetic and biochemical basis for alternative routes of tocotrienol biosynthesis for enhanced vitamin E antioxidant production," The Plant Journal, vol. 73, no. 4, pp. 628-639, 2013. 
[15] K. Biswas, V. Muthukkumarasamy, and W. L. Tan, "Blockchain based wine supply chain traceability system," Future Technologies Conference (FTC), Canada, Vancouver, pp. 56-62, 2017.

[16] N. Kamaruddin and A. Wahab, "Interlaboratory data fusion repository system (InDFuRS) for tocotrienols-based treatment," Indonesian Journal of Electrical Engineering and Computer Science, vol. 13, no. 13, pp. 1130-1135, 2019.

[17] P. Meganathan, R. S. Jabir, H. G. Fuang, N. Bhoo-Pathy, R. B. Choudhury, N. A. Taib, K. Nesaretnam, and Z. Chik, "A new formulation of gamma delta tocotrienol has superior bioavailability compared to existing tocotrienolrich fraction in healthy human subjects," Scientific Reports, vol. 5, pp. 13550, 2015.

[18] F. Tian, "An agri-food supply chain traceability system for China based on RFID \& blockchain technology," 2016 13th International Conference on Service Systems and Service Management (ICSSSM), Kunming, pp. 1-6, 2016.

[19] A. Litke, D. Anagnostopoulos, and T. Varvarigou, "Blockchains for supply chain management: Architectural elements and challenges towards a global scale deployment," Logistics, vol. 3, no. 1, pp. 1-17, 2019.

[20] R. Y. Chen, "A traceability chain algorithm for artificial neural networks using t-s fuzzy cognitive maps in blockchain," Future Generation Computer Systems, vol. 80, pp. 198-210, 2018.

[21] M. Benchoufi, R. Porcher, and P. Ravaud, "Blockchain Protocols in clinical trials: Transparency and traceability of consent," F1000 Research, vol. 6, no. 66, pp. 1-26, 2018.

[22] J. F. Galvez, J. C. Mejutob, and J. Simal-Gandarac, "Future challenges on the use of blockchain for food traceability analysis," TrAC Trends in Analytical Chemistry, vol. 107, pp. 222-232, October 2018.

[23] M. Risius and K. Spohrer, "A blockchain research framework," Business \& Information Systems Engineering, vol. 59, no. 6, pp 385-409, 2017.

[24] M. N. Saadat, S. A. Halim, H. Osman, R. M. Nassr, and M. F. Zuhairi, "Blockchain based crowdfunding systems," Indonesian Journal of Electrical Engineering and Computer Science, vol. 15, no. 1, pp. 409-413, July 2019.

[25] N. Kamaruddin and A. Wahab, "Features extraction for speech emotion," Journal of Computational Methods in Sciences and Engineering, vol. 9, no. 1, 2S1, pp. 1-12, 2009.

[26] H. S. Yaacob, A. Wahab, and N. Kamaruddin, "CMAC-based computational model of affects (CCMA) from self-organizing feature mapping weights for classification of emotion using EEG signals," International Journal for Computers \& Their Applications, vol. 22, no. 1, pp. 31-42, 2015.

[27] A. Abdulkareem, C. O. A. Awosope, and A. E. Tope-Ojo, "Development and implementation of a miniature RFID system in a shopping mall environment," International Journal of Electrical and Computer Engineering, vol. 9, no. 2, pp. 1374-1378, April 2019.

[28] M. M. Subashini, S. Das, S. Heble, U. Raj, and R. S. Karthik, "Internet of Things based wireless plant sensor for smart farming," Indonesian Journal of Electrical Engineering and Computer Science, vol. 10, no. 2, pp. 456-468, 2018.

[29] N. Kamaruddin, A. Wahab, A. Rahman, and D. Handiyani, "Pornography addiction detection based on neurophysiological computational approach," Indonesian Journal of Electrical Engineering and Computer Science, vol. 10, no. 1, pp. 138-145, 2018.

[30] N. Kamaruddin, A. Wahab, and Y. Rozaidi, "Neuro-physiological porn addiction detection using machine learning approach," Indonesian Journal of Electrical Engineering and Computer Science, vol. 16, no. 2, pp. 964-971, 2019.

[31] A. Wahab and N. Kamaruddin, "Brain developmental disorders' modelling based on preschoolers neuro-physiological profiling," Indonesian Journal of Electrical Engineering and Computer Science, vol. 12, no. 2, pp. 542-547, 2018.

\section{BIOGRAPHIES OF AUTHORS}



Assoc. Prof. Ts. Dr. Norhaslinda Kamaruddin currently holds a post of associate professor in Faculty of Computer and Mathematical Sciences, Universiti Teknologi MARA (UiTM), Malaysia. She served UiTM since 2011. She received her bachelor's degree in Information Technology (Computer Science) from Universiti Kebangsaan Malaysia in 2001 followed by her Master of Software Engineering from Malaya University in 2006. In 2013, she is awarded a Doctor of Philosophy (Computer Engineering) from Nanyang Technological University (Singapore) focusing on computational intelligence especially on Affective Computing. She is very active in the research fields of affective computing, speech emotion recognition, neurocognitive informatics, big data analytics, and driver behavioral study.

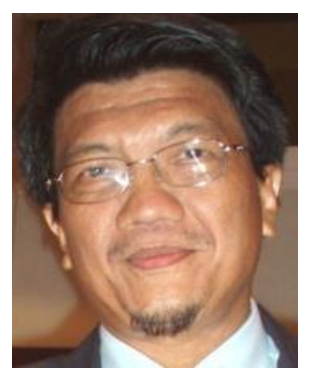

Prof. Dr. Abdul Wahab received the Degree from the University of Essex, Essex, U.K., in 1979, the M.Sc. degree from the National University of Singapore, Singapore, in 1987, and the Ph.D. degree from Nanyang Technological University, Singapore. His research has been in the areas of telecommunication, signal processing, and artificial intelligence. He was with Hewlett Packard Singapore, Singapore, as a Research and Development Project Manager both in CO, USA. He joined Nanyang Technological University in 1990, where he was an Associate Professor, before joining the International Islamic University of Malaysia, Malaysia, as a Professor, in 2009. He has authored over 100 conference papers, journal papers, patents, and book chapters in the areas of digital and optical computing, signal processing, and artificial intelligence. 